\title{
Future challenges for hematopoietic stem cell research
}

\author{
Gerald J. Spangrude
}

BioTechniques 35:1273-1279 (December 2003)

\begin{abstract}
This perspective summarizes several important advances in hematopoietic stem cell (HSC) biology in the past few years and places these advances in the context of future directions in stem cell research. The potential utility of stem cells for gene therapy, tissue engineering, and the treatment of neurological and other forms of disease is simply too significant to ignore, and yet our knowledge and ability to deliver these forms of therapy in a safe and efficacious manner will require additional advances in the understanding of the basic biology of stem cells.
\end{abstract}

\section{INTRODUCTION}

What will be the major topics for hematopoietic stem cell (HSC) research in the years to come? Ten years ago, several issues were hotly debated. What phenotype best characterizes HSC in the mouse (1)? How does this phenotype translate to man (2)? Are HSC sufficient to promote both rapid and sustained engraftment (3), or do progenitor cells play a major role in the latter process (4)? Is there a clinical advantage to transplantation of enriched cell populations compared to unseparated bone marrow (5)? What cytokines can stimulate HSC (6), and what cells produce these factors in vivo (7)? Once cell division begins, is the HSC fated to differentiate, or can it return to a quiescent state? Is this the mechanism of self-renewal? Why do HSC mobilize from the bone marrow (8), and why are mobilized cells superior to bone marrow harvests in promoting rapid engraftment of transplant recipients? Can expanded stem cell products be used to enhance engraftment in a transplant setting (9)?

Understanding HSC biology has historically been a retrospective exercise, in that one would evaluate the result of the culture or the transplant study in order to understand the potential of the cells that were initially in hand. With the development of methods to facilitate prospective isolation of HSC (10-12), many of the above questions have been successfully addressed. At the same time, many new questions have come under our scrutiny, questions that we simply did not know enough to even formulate 10 years ago. Are HSC highly fusagenic (13)? Does this property explain their ability to contribute genetic material to a diverse range of nonmesenchymal tissues (14)? Or are HSC a leftover remnant of a developmental program that includes an ability to dedifferentiate? In either case, can these unusual properties of fusion, plasticity, and transdifferentation be exploited to facilitate tissue repair or gene therapy? A recent review explores these issues in much greater detail (15).

\section{BASIC MECHANISMS OF HSC BIOLOGY}

In recent years, a surprising degree of contribution by essential developmental programs to the control of hematopoiesis has been demonstrated. The mammalian functions of these conserved developmental programs, many of which were originally described in the fruit fly model system, underscore the important contributions made by seemingly esoteric basic scientific investigation to our understanding of human development and pathology. The studies that initially established this bridge between developmental control in model organisms and mammalian hematopoiesis focused on the Hox family of genes, based on the role of some Hox family members in leukemic transformation. Expression analysis of several Hox family members within the hierarchy of human hematopoietic stem and progenitor cells revealed intriguing patterns of $H O X$ gene expression depending upon the specific Hox family member (16). In particular, HOXB4 was observed to have its highest level of expression at the most primitive stages of human hematopoietic development and to decrease as differentiation proceeded. In contrast, other members of the Hox family were up-regulated during the process of hematopoietic differentiation, but were extinguished during the final stages of hematopoietic development and were silent in mature cells. These observations led to the hypothesis that differential expression of Hox family members might be one mechanism by which hematopoietic self-renewal and differentiation could be regulated (17). This prediction was con- 
firmed for a number of Hox family members, particularly for HOXB4 and HOXA10. Enforced expression of HOXB4 leads to enhanced self-renewal of HSC in a number of contexts, including mouse and human models, which performed in vitro as well as in vivo $(18,19)$. The enhancement of self-renewing divisions is overcome with the onset of differentiation, leading to normal development of hematopoietic lineages in the absence of transformation. Furthermore, enforced expression of HOXB4 in mouse embryonic stem (ES) cells leads to enhanced hematopoietic differentiation (20) and allows ES cells to reconstitute hematopoiesis in a transplantation model, something that is not observed in the absence of HOXB4 expression (21). These were the first observations supporting the concept that genetic programs that guide embryonic development are retained in adult hematopoiesis and can regulate the hematopoietic process.

A second area of focus in the genetic control of hematopoiesis has been the Notch family of receptors and their ligands. Again, interest in these molecules originated in the discovery that translocations involving the Notch receptors were associated with human leukemias. Based on the hypothesis that activation of the Notch signaling pathway might regulate hematopoietic development, a number of laboratories utilized myeloid developmental model systems to establish a role for this pathway in hematopoietic self-renewal. However, a far more dramatic role for the Notch pathway in regulation of hematopoiesis has been demonstrated during the process of lymphoid lineage specification.

The Notch proteins are a family of transmembrane receptors, first described in the fruit fly as a regulator of wing morphology. In mammals, four genes encode a family of Notch receptors, which share similar structure and ligands but distinct patterns of expression (reviewed in Reference 22). First identified in humans in a chromosomal translocation associated with acute T-lymphocytic leukemia (23), NOTCHI has since been established as a critical component in the process of $\mathrm{T}$ cell development. This conclusion was first suggested by experiments in which the activated intracellular region of NOTCH1, when expressed in bone marrow cells, produced leukemias exclusively of the $\mathrm{T}$ lineage (24). Analysis of animals prior to the emergence of leukemia demonstrated that expression of activated NOTCHI leads to a block of B cell development and the emergence of immature T cells in the bone marrow, suggesting a role for $\mathrm{NOTCH} 1$ in the determination of $\mathrm{T}$ and B lineages (25). Targeted deletion of Notch1 resulted in embryonic lethality, however the generation of a conditional Notchl knock-out mouse strain allowed the analysis of a loss of function mutation beyond embryonic development. These experiments demonstrated that targeted deletion of Notchl resulted in a block in T cell development, accompanied by the presence of B lymphocytes in the thymus (26). Recent new studies have extended these initial observations. One study has demonstrated that the failure of $\mathrm{T}$ lineage development in Notch1-deficient animals is not due to a defect in the homing of progenitors to the thymus. In the absence of Notchl signaling, the developmental outcome of progenitors that home to the thymus is that of a default progression down the B cell lineage (27). Several conditional knock-out models have allowed analysis of Notchl function at discrete stages of
$\mathrm{T}$ cell development. These studies revealed that conditional deletion of Notchl at an early stage of T cell development results in a severe impairment in the generation of the $\alpha \beta$ lineage of T cells, while the $\gamma \delta$ lineage develops normally in the absence of Notchl (28). However, conditional mutation of Notchl after the process of $\mathrm{T}$ cell receptor rearrangement has been completed showed no further effect of the loss of Notchl on T cell differentiation or function (29). These genetic disruption studies contrast with previous results obtained with misexpression approaches, suggesting that in addition to NOTCH1, other members of the Notch family of receptors may also play important roles in later stages of $\mathrm{T}$ cell development (30).

An additional level of control over Notch signaling in lymphocyte development has been demonstrated at the level of multiple ligands. Using a cell co-culture assay, in which the Notch ligands Delta-1 and Jagged-1 were expressed by stromal cells, Jaleco et al. showed differential effects induced by these ligands on human lymphoid differentiation (31). Delta1 promoted T/NK cell development while inhibiting B lymphocyte commitment from human CD34+ progenitor cells in vitro. In this culture model, Delta- 1 induced CD4, CD8, and cytoplasmic CD3 expression, suggesting a significant role for this Notch1 ligand in T cell development. In contrast, Jagged1 did not disturb B lineage outgrowth or support CD3, CD4, or CD8 expression. These results have subsequently been extended in a mouse model, in which expression of Delta-like-1 by OP9 stromal cells promoted differentiation of hematopoietic progenitors into CD4 CD8 double- and single-positive $\mathrm{T}$ cells (32). This observation represents a major advance in the field, establishing that stimulation of Notch receptors by Delta-like-1 provides key signals for $\mathrm{T}$ cell differentiation in the absence of an intact thymus.

Further regulation of the Notch signaling pathway in lymphopoiesis can be traced to the intracellular events that occur prior to or after Notch stimulation by a ligand. Research in two laboratories has yielded data supporting roles for modulators of Notch in lymphoid lineage specification. Two negative regulators of Notchl, Lunatic Fringe and Deltexl, induced lymphoid progenitors to differentiate into B cells in the thymus when ectopically expressed $(33,34)$. Another inhibitor of Notch signaling, Numb, lacked any effect on T cell development (35). These data show that both inductive and inhibitory signals in the Notchl signaling pathway are central components in the mechanism of $\mathrm{T}$ and $\mathrm{B}$ lineage commitment. In contrast, the inhibition of B cell development in cells expressing activated Notch proteins may be mediated by the ability of the HES1 and HES5 proteins, which are induced by Notch, to antagonize the E2A transcription factor (36). NOTCHI overexpression has also been shown to influence lymphoid differentiation in a non-cell autonomous manner. Cells transduced with activated $\mathrm{NOTCH} 1$ produced a soluble factor that influenced neighboring cells to repress B lineage development and promote myeloid development, both in vitro and in vivo (37). The conclusions reached by these recent studies clearly indicate a major role for Notch signaling in the regulation of early lymphoid differentiation $(38,39)$.

Very recently, a third developmentally important pathway has been demonstrated to be influential in the regulation of 
hematopoietic self-renewal. Originally described as a frequent target for insertional mutagenesis by mouse mammary tumor viruses (40), the family of Wnt proteins has subsequently been shown to play important roles in animal development including regulation of stem cell proliferation (41). However, difficulties in purifying active soluble forms of Wnt family members have prevented many functional studies. This problem was recently overcome with the discovery that Wnt proteins are lipid-modified and as such require hydrophobic conditions for isolation and purification (42). Utilizing this newly available reagent, it has been demonstrated directly that culture of mouse HSC in the presence of soluble Wnt3a protein and low concentrations of Steel factor leads to proliferation of HSC with maintenance of HSC phenotype and function. This effect can be reproduced by overexpression of downstream members of the Wnt signaling pathway and inhibited by blocking the pathway (43). Interestingly, both HoxB4 and Notchl are up-regulated in HSC overexpressing activated $\beta$-catenin, a downstream mediator of Wnt signaling. These observations suggest that Wnt signaling is central to the process of HSC self-renewal and provides a means by which normal HSC might be expanded in an in vitro culture system to allow subsequent transplantation in a clinical setting. While such applications could be envisioned based on expression of HoxB4 or Notch family members, the obvious advantage of stimulation by a soluble protein compared to the need to express intracellular transcription factors such as Notch or HoxB4 makes clinical application much more feasible. Whether these exciting new discoveries mark the dawn of a new age for cellular therapy remains to be seen. At the least, the future of ex vivo expansion of HSC for transplantation purposes seems brighter than ever before due to these new findings.

\section{GENE THERAPY}

While the future may be bright for ex vivo expansion, gene therapists have suffered a number of severe setbacks in the past few years. The use of extraordinary large doses of an adenovirus-based gene therapy vector led to the death of a patient enrolled in an experimental study, raising troubling questions about the risk-benefit ratios of specific approaches to gene therapy (44). More troubling still is the concern that the clinical judgment of physicians who conduct these types of studies might be sufficiently influenced by potential monetary or career rewards to obscure questions of patient safety (45). A third concern, one that had been repeatedly discussed and ultimately discarded as being very unlikely to influence clinical outcome, is insertional mutagenesis resulting from the genomic integration of retroviral gene therapy vectors. Since such integration occurs randomly, and since so much of the human genome consists of untranslated sequences, it follows that the chance of a retrovirus interrupting a crucial gene or activating a proto-oncogene would be quite small. The field was, therefore, shocked to learn that two of eleven patients treated for X-linked severe combined immunodeficiency (SCID-X1) by retroviral gene therapy subsequently developed T cell leukemia (46). This initial shock was com- pounded when it was discovered that the leukemia in both patients resulted from integration of the vector into the same gene, LMO2 (47). This decidedly nonrandom outcome of insertional mutagenesis raises concerns about the future of gene therapy at its current state of development.

Recalling the first applications of retrovirally based gene therapy in humans, one must wonder how the SCID-X1 trial showed such a remarkable success followed by such a tragic outcome. The first attempts to transduce human cells prior to transplantation showed rather low transduction efficiencies, both with proliferating lymphocytes (48) and with cord blood hematopoietic cells (49) as targets. Improvements in vector design and transduction protocols undoubtedly contributed to improvements in gene transduction over the years. However, one cannot help but consider the possibility that both the success and the failure of the SCID-X1 trial may be related to the power of biological selection in this application. SCID-X1 patients lack a functional $\gamma$ chain that interacts with other proteins to form receptors for at least three cytokines that function during lymphoid development. In the absence of the proliferative and differentiative signals delivered through these receptors, lymphoid development fails (50). Provision of the $\gamma$ chain by retroviral transduction rescues this defect, and cells that successfully express the transgene after transduction have a tremendous proliferative advantage over those that do not express it. This leads to selective outgrowth of transduced cells and correction of the immunological defect. In this way, the SCID-X1 trial differs fundamentally from earlier trials in adenosine deaminase (ADA)-deficient patients, in which the selective advantage imparted to transduced cells was of a detoxifying rather than a proliferative nature. Further, ADA patients continued to receive supplementary ADA, which likely would blunt any selective effect of the gene transduction. The strong selective pressure imparted by restoration of a proliferative response in SCID-X1 patients likely explains the more profound clinical effect seen in this trial. Could a similarly strong selection also be the reason that activation of $L M O 2$ by insertional mutagenesis has been seen in two of eleven patients? If LMO2 plays a central role in early lymphoid development, combined expression of the $\gamma$ chain with LMO2 may lead to synergistic proliferative effects that would result in stronger selection. The fate of this form of gene therapy remains to be seen. If the T cell leukemia responds well to therapy in all cases, the risk of this therapeutic approach may be acceptable in comparison to the potential benefit to the patient.

\section{CANCER STEM CELLS}

As discussed earlier in the context of developmental programs that influence hematopoiesis, the field of HSC biology has benefited greatly from lessons learned from leukemias and other human diseases of the blood. A parallel to this process of lateral translation of knowledge from seemingly unrelated fields is now emerging with the realization that the ability of HSC to undergo a process of self-renewal may be relevant not only to leukemias but to the study of all types of neoplasia (51). The concept of a stem cell origin of leukemia 
has evolved from the classical view that leukemias represent an arrested state of development in which the differentiation process has halted but proliferation continues. However, the realization that only a small fraction of leukemic cells demonstrated clonogenicity in culture suggested that only a subset of the leukemic cells was responsible for maintaining the leukemia by clonal expansion. Cell isolation experiments confirmed this hypothesis and demonstrated that the leukemia-initiating cell, in most cases, could be found among phenotypically immature cells that resembled HSC (52). A second series of experiments showed that chromosomal translocations leading to transformation can persist in the HSC compartment, only to lead to transformation at the progenitor stage of hematopoietic development (53). Thus, both stem and progenitor cells can serve as targets of transformation, possibly after the accumulation of additional oncogenic genetic alterations (54).

In retrospect, the concept of a stem or progenitor cell basis for leukemia is far more satisfying that the long-standing theory that leukemias arise due to transformation at any point from the most primitive blast cells to the more mature differentiated stages of development. Stem cells are present continually in tissues over the long periods of time that would be expected to be necessary for the accumulation of genetic alterations. Due to their high intrinsic proliferation potential, stem cells produce large numbers of progeny, including other stem cells produced through self-renewal. This provides an expanded and self-renewing target population for mutation that could not be duplicated at later stages of development when proliferation is low. Finally, the process of self-renewal in HSC could be subverted to promote transformation far more easily than at a stage of development where self-renewal is no longer occurring. Excessive self-renewal of stem cells would allow the accumulation of more mutations, thus increasing the chances of transformation. Alternatively, progenitor cells that fail to limit their self-renewal (e.g., by mutation of the Wnt pathway or aberrant expression of $\mathrm{HOXB4}$ ) may also go on to transformation. Thus, although common pathways have been demonstrated to regulate both stem cell self-renewal and oncogenesis, the target cells for transformation may include progenitors as well as stem cells.

The concept of a stem cell origin for neoplasia has recently been extended to solid tumors with the identification of human mammary stem/progenitor cells and the demonstration that these cells, but not the majority of breast cancer cells, can form new tumors in an animal model (55). Isolation of tumor stem cells strongly suggests that within the tumor mass, only a small definable subset of cells possess the characteristics necessary to allow sufficient proliferation to form a tumor. These characteristics must be lost during the course of tumor cell expansion, since the majority of the tumor cells fail to proliferate. It is therefore fully consistent with the function of stem cells in normal tissues, where self-renewal of a small subset of cells rather than proliferation of mature cells is central to the maintenance of the tissue. The important implication underlying these observations is that in order to be maximally effective, therapeutic management of neoplasia must target the tumor stem cells rather than the majority of the tumor mass (51). To do this, we must more fully under- stand the characteristics of both normal and cancerous stem cells in order to fully exploit potential avenues for therapy. Such characteristics include high proliferation potential (selfrenewal) coupled with a slow cell cycle (extended $G_{0}$ or $G_{1}$ phases), expression of drug efflux pumps and anti-apoptotic proteins, and in the case of some leukemias, shared cell surface antigen expression. Expression profiling approaches promise to provide exceptionally powerful tools for diagnosis and prospective therapy of cancer (56). These approaches will also be valuable for the analysis of normal hematopoiesis as discussed by Akashi and Li elsewhere in this issue (p. 1233).

\section{EMBRYONIC STEM CELLS TO MODEL HSC DEVELOPMENT}

A final area of future development that will involve HSC is the emerging field of human ES cell biology. Working from the platform established through years of mouse ES cell studies (57-59), it is now reasonable to contemplate human in vitro models for the specification of HSC in development, the regulation of early blood progenitors, and the differentiation of human lymphoid lineages for which previous model systems failed to fully recapitulate. Evidence of these kinds of studies are now appearing in the literature (60), and it seems very likely that with the renewed commitment of federal agencies to promote human ES cell research through increased funding (61), hematology researchers will be in a very good position to further the understanding of the molecular underpinnings of human hematopoiesis. Dr. Kaufman and coworkers provides a much more detailed description of this area of research elsewhere in this issue (p. 1253).

\section{CONCLUSION}

In the 1980s, immunologists experienced a professional transition in which their area of research interest moved from behind the shadow of microbiology and into the public eye. The recognition that acquired immunodeficiency syndrome (AIDS) was caused by a virus that attacks the immune system caused public interest in immunology to skyrocket; one could discuss the CD4 T cell on a public bus with a stranger. Today, a similar phenomenon is unfolding for stem cell biologists, as ethicists, celebrities, and pundits join scientists in debate over the experimental and clinical use of human ES cells. As the premier example of clinically relevant stem cells, HSC will certainly play a central role in guiding future applications of stem cell therapy. What other developments might we predict for the future of HSC biology? As retrospective as science can be, one must conclude that only time will tell.

\section{REFERENCES}

1.Orlic, D. and D.M. Bodine. 1994. What defines a pluripotent hematopoietic stem cell (PHSC): will the real PHSC please stand up! Blood 84: 3991-3994.

2.Baum, C.M., I.L. Weissman, A.S. Tsukamoto, A. Buckle, and B. Peault. 1992. Isolation of a candidate human hematopoietic stem-cell population. 
Proc. Natl. Acad. Sci. USA 89:2804-2808.

3.Zijlmans, J.M., J.W. Visser, L. Laterveer, K. Kleiverda, D.P. Heemskerk, P.M. Kluin, R. Willemze, and W.E. Fibbe. 1998. The early phase of engraftment after murine blood cell transplantation is mediated by hematopoietic stem cells. Proc. Natl. Acad. Sci. USA 95:725-729.

4.Jones, R.J., P. Celano, S.J. Sharkis, and L.L. Sensenbrenner. 1989. Two phases of engraftment established by serial bone marrow transplantation in mice. Blood 73:397-401.

5.Brugger, W., R. Henschler, S. Heimfeld, R.J. Berenson, R. Mertelsmann, and L. Kanz. 1994. Positively selected autologous blood CD34+ cells and unseparated peripheral blood progenitor cells mediate identical hematopoietic engraftment after high-dose VP16, ifosfamide, carboplatin, and epirubicin. Blood 84:1421-1426.

6.Li, C.L. and G.R. Johnson. 1992. Rhodamine123 reveals heterogeneity within murine Lin-, Sca-1+ hemopoietic stem cells. J. Exp. Med. 175 1443-1447.

7.Ogawa, M., Y. Matsuzaki, S. Nishikawa, S. Hayashi, T. Kunisada, T. Sudo, T. Kina, and H. Nakauchi. 1991. Expression and function of c-kit in hemopoietic progenitor cells. J. Exp. Med. 174:63-71.

8.Papayannopoulou, T., G.V. Priestley, and B. Nakamoto. 1998. AntiVLA4/VCAM-1-induced mobilization requires cooperative signaling through the kit/mkit ligand pathway. Blood 91:2231-2239.

9.Brugger, W., S. Heimfeld, R.J. Berenson, R. Mertelsmann, and L. Kanz. 1995. Reconstitution of hematopoiesis after high-dose chemotherapy by autologous progenitor cells generated ex vivo. N. Engl. J. Med. 333:283-287.

10.Visser, J.W.M., J.G.J. Bauman, A.H. Mulder, J.F. Eliason, and A.M. de Leeuw. 1984. Isolation of murine pluripotent hemopoietic stem cells. J. Exp. Med. 159:1576-1590.

11.Spangrude, G.J., S. Heimfeld, and I.L. Weissman. 1988. Purification and characterization of mouse hematopoietic stem cells. Science 241:58-62.

12.Goodell, M.A., K. Brose, G. Paradis, A.S. Conner, and R.C. Mulligan. 1996. Isolation and functional properties of murine hematopoietic stem cells that are replicating in vivo. J. Exp. Med. 183:1797-1806.

13.Wang, X., H. Willenbring, Y. Akkari, Y. Torimaru, M. Foster, M. AlDhalimy, E. Lagasse, M. Finegold, et al. 2003. Cell fusion is the principal source of bone-marrow-derived hepatocytes. Nature 422:897-901.

14.Krause, D.S., N.D. Theise, M.I. Collector, O. Henegariu, S. Hwang, R. Gardner, S. Neutzel, and S.J. Sharkis. 2001. Multi-organ, multi-lineage engraftment by a single bone marrow-derived stem cell. Cell 105:369-377.

15.Herzog, E.L., L. Chai, and D.S. Krause. 2003. Plasticity of marrow derived stem cells. Blood, 1st ed. paper, prepublished online July 31, 2003; DOI 10.1182/blood-2003-05-1664.

16.Sauvageau, G., P.M. Lansdorp, C.J. Eaves, D.E. Hogge, W.H. Dragowska, D.S. Reid, C. Largman, H.J. Lawrence, and R.K. Humphries. 1994. Differential expression of homeobox genes in functionally distinct CD34+ subpopulations of human bone marrow cells. Proc. Natl. Acad. Sci. USA 91:12223-12227.

17.Sauvageau, G., U. Thorsteinsdottir, C.J. Eaves, H.J. Lawrence, C. Largman, P.M. Lansdorp, and R.K. Humphries. 1995. Overexpression of HOXB4 in hematopoietic cells causes the selective expansion of more primitive populations in vitro and in vivo. Genes Dev. 9:1753-1765.

18.Antonchuk, J., G. Sauvageau, and R.K. Humphries. 2002. HOXB4-induced expansion of adult hematopoietic stem cells ex vivo. Cell 109:39-45.

19.Antonchuk, J., G. Sauvageau, and R.K. Humphries. 2001. HOXB4 overexpression mediates very rapid stem cell regeneration and competitive hematopoietic repopulation. Exp. Hematol. 29:1125-1134.

20.Helgason, C.D., G. Sauvageau, H.J. Lawrence, C. Largman, and R.K. Humphries. 1996. Overexpression of HOXB4 enhances the hematopoietic potential of embryonic stem cells differentiated in vitro. Blood 87:27402749.

21.Kyba, M., R.C. Perlingeiro, and G.Q. Daley. 2002. HoxB4 confers definitive lymphoid-myeloid engraftment potential on embryonic stem cell and yolk sac hematopoietic progenitors. Cell 109:29-37.

22.Allman, D., J.A. Punt, D.J. Izon, J.C. Aster, and W.S. Pear. 2002. An invitation to T and more: notch signaling in lymphopoiesis. Cell 109(Suppl): S1-S11.

23.Ellisen, L.W., J. Bird, D.C. West, A.L. Soreng, T.C. Reynolds, S.D. Smith, and J. Sklar. 1991. TAN-1, the human homolog of the Drosophila notch gene, is broken by chromosomal translocations in $\mathrm{T}$ lymphoblastic neoplasms. Cell 66:649-661.

24.Pear, W.S., J.C. Aster, M.L. Scott, R.P. Hasserjian, B. Soffer, J. Sklar, and D. Baltimore. 1996. Exclusive development of T cell neoplasms in mice transplanted with bone marrow expressing activated Notch alleles. J. Exp. Med. 183:2283-2291.

25.Pui, J.C., D. Allman, L. Xu, S. DeRocco, F.G. Karnell, S. Bakkour, J.Y. Lee, T. Kadesch, et al. 1999. Notch1 expression in early lymphopoiesis influences B versus T lineage determination. Immunity 11:299-308.

26.Radtke, F., A. Wilson, G. Stark, M. Bauer, J. van Meerwijk, H.R. MacDonald, and M. Aguet. 1999. Deficient T cell fate specification in mice with an induced inactivation of Notch1. Immunity 10:547-558

27.Wilson, A., H.R. MacDonald, and F. Radtke. 2001. Notch 1-deficient common lymphoid precursors adopt a B cell fate in the thymus. J. Exp. Med. 194:1003-1012.

28.Wolfer, A., A. Wilson, M. Nemir, H.R. MacDonald, and F. Radtke. 2002. Inactivation of Notch1 impairs VDJbeta rearrangement and allows pre-TCR-independent survival of early alpha beta lineage thymocytes. Immunity 16:869-879.

29.Wolfer, A., T. Bakker, A. Wilson, M. Nicolas, V. Ioannidis, D.R. Littman, P.P. Lee, C.B. Wilson, et al. 2001. Inactivation of Notch 1 in immature thymocytes does not perturb CD4 or CD8T cell development. Nat. Immunol. 2:235-241.

30.Rothenberg, E.V. 2001. Notchless T cell maturation? Nat. Immunol. 2: 189-190.

31.Jaleco, A.C., H. Neves, E. Hooijberg, P. Gameiro, N. Clode, M. Haury, D. Henrique, and L. Parreira. 2001. Differential effects of Notch ligands Delta-1 and Jagged-1 in human lymphoid differentiation. J. Exp. Med. 194: 991-1002.

32.Schmitt, T.M. and J.C. Zuniga-Pflucker. 2002. Induction of T cell development from hematopoietic progenitor cells by delta-like-1 in vitro. Immunity 17:749-756.

33.Koch, U., T.A. Lacombe, D. Holland, J.L. Bowman, B.L. Cohen, S.E. Egan, and C.J. Guidos. 2001. Subversion of the T/B lineage decision in the thymus by lunatic fringe-mediated inhibition of Notch-1. Immunity 15 : 225-236.

34.Izon, D.J., J.C. Aster, Y. He, A. Weng, F.G. Karnell, V. Patriub, L. Xu, S. Bakkour, et al. 2002. Deltex1 redirects lymphoid progenitors to the B cell lineage by antagonizing Notch1. Immunity 16:231-243.

35.French, M.B., U. Koch, R.E. Shaye, M.A. McGill, S.E. Dho, C.J. Guidos, and C.J. McGlade. 2002. Transgenic expression of numb inhibits notch signaling in immature thymocytes but does not alter $\mathrm{T}$ cell fate specification. J. Immunol. 168:3173-3180.

36.Kawamata, S., C. Du, K. Li, and C. Lavau. 2002. Overexpression of the Notch target genes Hes in vivo induces lymphoid and myeloid alterations. Oncogene 21:3855-3863.

37.Kawamata, S., C. Du, K. Li, and C. Lavau. 2002. Notch1 perturbation of hemopoiesis involves non-cell-autonomous modifications. J. Immunol. 168:1738-1745.

38.Radtke, F., A. Wilson, B. Ernst, and H.R. MacDonald. 2002. The role of Notch signaling during hematopoietic lineage commitment. Immunol. Rev. 187:65-74.

39.Allman, D., J.C. Aster, and W.S. Pear. 2002. Notch signaling in hematopoiesis and early lymphocyte development. Immunol. Rev. 187:75-86.

40.Nusse, R., A. Brown, J. Papkoff, P. Scambler, G. Shackleford, A. McMahon, R. Moon, and H. Varmus. 1991. A new nomenclature for int-1 and related genes: the Wnt gene family. Cell 64:231.

41.Cadigan, K.M. and R. Nusse. 1997. Wnt signaling: a common theme in animal development. Genes Dev. 11:3286-3305.

42.Willert, K., J.D. Brown, E. Danenberg, A.W. Duncan, I.L. Weissman, T. Reya, J.R. Yates III, and R. Nusse. 2003. Wnt proteins are lipid-modified and can act as stem cell growth factors. Nature 423:448-452.

43.Reya, T., A.W. Duncan, L. Ailles, J. Domen, D.C. Scherer, K. Willert, L. Hintz, R. Nusse, and I.L. Weissman. 2003. A role for Wnt signalling in self-renewal of haematopoietic stem cells. Nature 423:409-414.

44.Bostanci, A. 2002. Gene therapy. Blood test flags agent in death of Penn subject. Science 295:604-605.

45.Lorman, A.J. 2001. Clinical trials face heightened scrutiny as science and commerce appear to merge. J. Biolaw Bus. 4:23-32

46.Hacein-Bey-Abina, S., C. von Kalle, M. Schmidt, F. Le Deist, N. Wulffraat, E. McIntyre, I. Radford, J.L. Villeval, et al. 2003. A serious adverse event after successful gene therapy for X-linked severe combined immunodeficiency. N. Engl. J. Med. 348:255-256.

47.Hacein-Bey-Abina, S., C. Von Kalle, M. Schmidt, M.P. McCormack, N. Wulffraat, P. Leboulch, A. Lim, C.S. Osborne, et al. 2003. LM02associated clonal $\mathrm{T}$ cell proliferation in two patients after gene therapy for 
SCID-X1. Science 302:415-419.

48.Culver, K.W., W.R. Osborne, A.D. Miller, T.A. Fleisher, M. Berger, W.F. Anderson. and R.M. Blaese. 1991. Correction of ADA deficiency in human T lymphocytes using retroviral-mediated gene transfer. Transplant. Proc. 23:170-171.

49.Schmidt, M., D.A. Carbonaro, C. Speckmann, M. Wissler, J. Bohnsack, M. Elder, B.J. Aronow, J.A. Nolta, et al. 2003. Clonality analysis after retroviral-mediated gene transfer to CD34+ cells from the cord blood of ADA-deficient SCID neonates. Nat. Med. 9:463-468.

50.Puck, J.M., S.M. Deschenes, J.C. Porter, A.S. Dutra, C.J. Brown, H.F. Willard, and P.S. Henthorn. 1993. The interleukin-2 receptor gamma chain maps to Xq13.1 and is mutated in X-linked severe combined immunodeficiency, SCIDX1. Hum. Mol. Genet. 2:1099-1104.

51.Reya, T., S.J. Morrison, M.F. Clarke, and I.L. Weissman. 2001. Stem cells, cancer, and cancer stem cells. Nature 414:105-111.

52.Bonnet, D. and J.E. Dick. 1997. Human acute myeloid leukemia is organized as a hierarchy that originates from a primitive hematopoietic cell. Nat. Med. 3:730-737.

53.Miyamoto, T., I.L. Weissman, and K. Akashi. 2000. AML1/ETO-expressing nonleukemic stem cells in acute myelogenous leukemia with 8;21 chromosomal translocation. Proc. Natl. Acad. Sci. USA 97:7521-7526.

54.Yuan, Y., L. Zhou, T. Miyamoto, H. Iwasaki, N. Harakawa, C.J. Hetherington, S.A. Burel, E. Lagasse, et al. 2001. AML1-ETO expression is directly involved in the development of acute myeloid leukemia in the presence of additional mutations. Proc. Natl. Acad. Sci. USA 98:10398-10403.

55.Al-Hajj, M., M.S. Wicha, A. Benito-Hernandez, S.J. Morrison, and M.F. Clarke. 2003. Prospective identification of tumorigenic breast cancer cells. Proc. Natl. Acad. Sci. USA 100:3983-3988.

56.Davis, R.E. and L.M. Staudt. 2002. Molecular diagnosis of lymphoid malignancies by gene expression profiling. Curr. Opin. Hematol. 9:333-338.

57.Daley, G.Q. 2003. From embryos to embryoid bodies: generating blood from embryonic stem cells. Ann. NY Acad. Sci. 996:122-131.

58.Mizuseki, K., T. Sakamoto, K. Watanabe, K. Muguruma, M. Ikeya, A. Nishiyama, A. Arakawa, H. Suemori, et al. 2003. Generation of neura crest-derived peripheral neurons and floor plate cells from mouse and primate embryonic stem cells. Proc. Natl. Acad. Sci. USA 100:5828-5833.

59.Yamamoto, H., G. Quinn, A. Asari, H. Yamanokuchi, T. Teratani, M. Terada, and T. Ochiya. 2003. Differentiation of embryonic stem cells into hepatocytes: biological functions and therapeutic application. Hepatology 37:983-993.

60.Chadwick, K., L. Wang, L. Li, P. Menendez, B. Murdoch, A. Rouleau, and M. Bhatia. 2003. Cytokines and BMP-4 promote hematopoietic differentiation of human embryonic stem cells. Blood 102:906-915.

61.Zerhouni, E. 2003. Embryonic stem cells. Stem cell programs. Science 300:911-912.

\section{Address correspondence to:}

Gerald J. Spangrude

Departments of Medicine and Pathology

University of Utah School of Medicine RM 4C416

30 N 1900 E

Salt Lake City, UT 84132-2408, USA

e-mail: gspangrude@mac.com 